\title{
Local Linearization-Runge Kutta (LLRK) Methods for Solving Ordinary Differential Equations
}

\author{
H. De la Cruz ${ }^{1,2}$, R.J. Biscay ${ }^{3}$, F. Carbonell ${ }^{3}$, \\ J.C. Jimenez ${ }^{3}$, and T. Ozaki ${ }^{4}$ \\ ${ }^{1}$ Universidad de Granma, Bayamo MN, Cuba \\ ${ }^{2}$ Universidad de las Ciencias Informáticas, \\ La Habana, Cuba \\ ${ }^{3}$ Instituto de Cibernética, Matemática y Física, \\ La Habana, Cuba \\ ${ }^{4}$ Institute of Statistical Mathematics, \\ Tokyo, Japan \\ hugo@uci.cu, biscay@icmf.inf.cu
}

\begin{abstract}
A new class of stable methods for solving ordinary differential equations (ODEs) is introduced. This is based on combining the Local Linearization (LL) integrator with other extant discretization methods. For this, an auxiliary ODE is solved to determine a correction term that is added to the LL approximation. In particular, combining the LL method with (explicit) Runge Kutta integrators yields what we call LLRK methods. This permits to improve the order of convergence of the LL method without loss of its stability properties. The performance of the proposed integrators is illustrated through computer simulations.
\end{abstract}

\section{Introduction}

The Local Linearization (LL) method (see, e.g., 9, 11, 15, 16, and references therein), also called exponentially fitted method [9], matricial exponentially fitted method [6], exponential Euler method [4] and piece-wise linearized method [16, is an explicit one-step integrator for solving ODEs. It is derived from the local linearization (first-order Taylor expansion) of the vector field of the equation at each time step. Theoretical and simulation results have demonstrated a number of dynamical properties of this approach, including A-stability and correct reproduction of phase portraits near hyperbolic equilibrium points and cycles [11. Furthermore, its computational cost is relatively low, even for systems that when deal with standard methods require the use of either cumbersome implicit schemes or extremely small time steps.

However, a major drawback of the LL method is its low order of convergence, namely two. This has motivated the recent development of a class of higher order LL methods (called LLT integrators) that add a Taylor-based correction term to the LL solution [3. In this way an arbitrary order of convergence is reached 
without losing the stability properties of the LL method. But this is achieved at the expense of computing higher order derivatives of the vector field of the equation.

In the present paper an alternative approach is introduced for constructing higher order LL integrators. It is also based on the addition of a correction term to the LL approximation, but now this is determined as the solution of an auxiliary ODE. For the latter, any discretization scheme can be used; e.g. an explicit Runge-Kutta (RK) method, leading to what we call the Local LinearizationRunge Kutta (LLRK) methods. Computation of higher order derivatives is not required, and A-stability is insured. This approach can be thought of as a flexible framework for increasing the order of the LL solution as well as for stabilizing standard explicit integrators.

Likewise splitting and Implicit-Explicit Runge-Kutta (IMEX RK) methods (see, e.g., [1], 13]), the LLRK method is based on the representation of the vector field as the addition of two components. However, there are notable differences between these approaches: i) Typically in splitting and IMEX methods such a decomposition is global instead of local, and it is not based on a first-order Taylor expansion. ii) In contrast with IMEX and LLRK approaches, splitting methods construct an approximate solution by composition of the flows corresponding to the component vector fields. iii) IMEX RK methods are partitioned (more specifically, additive) Runge-Kutta methods that compute a solution $\mathbf{y}=\mathbf{u}+\mathbf{z}$ by solving certain ODE for $(\mathbf{u}, \mathbf{z})$, setting different RK coefficients for each block. LLRK methods also solve a partitioned system for $(\mathbf{u}, \mathbf{z})$ but a different one. In this, one of the blocks is linear and uncouple, and it is solved by the LL discretization. After inserting the (continuous time) LL approximation into the second block, this is treated as a non-autonomous ODE, for which any extant RK discretization can be used.

The paper is organized as follows. Section 2 reviews the standard, low order LL method, and its numerical implementation. Section 3 introduces a new class of higher order LL methods, including the Local Linearization-Runge Kutta (LLRK) methods, and discussed its computational aspects. Finally, Section 4 illustrates its performance through computer simulations.

\section{Local Linearization Method}

Let $\mathcal{D} \subset \mathbb{R}^{d}$ be an open set and $\mathbf{f} \in C^{1}\left(\mathbb{R} \times \mathcal{D}, \mathbb{R}^{d}\right)$. Consider the initial-value problem

$$
\mathbf{x}^{\prime}(t)=\mathbf{f}(t, \mathbf{x}(t)), \quad t_{0} \leq t \leq T, \quad \mathbf{x}\left(t_{0}\right)=\mathbf{x}_{0},
$$

where $t_{0}, T \in \mathbb{R}, \mathbf{x}_{0} \in \mathcal{D}$. Let $t_{0}<t_{1}<\ldots<t_{N}=T$ be a given partition of $\left[t_{0}, T\right]$, and denote $h_{n}=t_{n+1}-t_{n}$ and $\boldsymbol{\Lambda}_{n}=\left[t_{n}, t_{n+1}\right]$ for $n=0, \ldots, N-1$.

The LL discretization can be derived as follows (see, e.g., [11]). Define the local problems

$$
\begin{aligned}
\mathbf{x}^{\prime}(t) & =\mathbf{f}(t, \mathbf{x}(t)), \quad t \in \boldsymbol{\Lambda}_{n} \\
\mathbf{x}\left(t_{n}\right) & =\mathbf{x}_{t_{n}},
\end{aligned}
$$


for given constants $\mathbf{x}_{t_{n}} \in \mathcal{D}, n=0, \ldots, N-1$. These equations are approximated by linear ones on the basis of the first-order Taylor expansion of $\mathbf{f}(t, \mathbf{x})$ around $\left(t_{n}, \mathbf{x}_{t_{n}}\right)$ :

$$
\mathbf{x}^{\prime}(t)=\mathbf{L}\left(t_{n}, \mathbf{x}_{t_{n}}\right) \mathbf{x}(t)+\mathbf{a}\left(t ; t_{n}, \mathbf{x}_{t_{n}}\right), \quad t \in \Lambda_{n}, \quad \mathbf{x}\left(t_{n}\right)=\mathbf{x}_{t_{n}},
$$

where

$$
\mathbf{L}(s, \mathbf{x})=\mathbf{f}_{x}^{\prime}(s, \mathbf{x}), \quad \mathbf{a}(t ; s, \mathbf{x})=\mathbf{f}(s, \mathbf{x})-\mathbf{f}_{x}^{\prime}(s, \mathbf{x}) \mathbf{x}+\mathbf{f}_{t}^{\prime}(s, \mathbf{x})(t-s) .
$$

Here, $\mathbf{f}_{x}^{\prime}$ and $\mathbf{f}_{t}^{\prime}$ denote the partial derivatives of $\mathbf{f}$ with respect to the variables $\mathbf{x}$ and $t$, respectively.

The problem (2) has a solution $\mathbf{y}^{L L}\left(. ; t_{n}, \mathbf{x}_{t_{n}}\right)$ that is explicit in terms of the fundamental matrix $\boldsymbol{\Phi}\left(t ; t_{n}, \mathbf{x}_{t_{n}}\right)=\exp \left(\left(t-t_{n}\right) \mathbf{f}_{x}^{\prime}\left(t_{n}, \mathbf{x}_{t_{n}}\right)\right)$ of the corresponding homogeneous linear system. Namely, for $t \in \boldsymbol{\Lambda}_{n}$,

$$
\begin{aligned}
\mathbf{y}^{L L}\left(t ; t_{n}, \mathbf{x}_{t_{n}}\right) & =\boldsymbol{\Phi}\left(t ; t_{n}, \mathbf{x}_{t_{n}}\right) \mathbf{x}_{t_{n}}+\int_{t_{n}}^{t} \mathbf{\Phi}\left(u ; t_{n}, \mathbf{x}_{t_{n}}\right) \boldsymbol{\Phi}^{-1}\left(u ; t_{n}, \mathbf{x}_{t_{n}}\right) \mathbf{a}\left(u ; t_{n}, \mathbf{x}_{t_{n}}\right) d u \\
& =\mathbf{x}_{t_{n}}+\left(t-t_{n}\right) \varphi\left(t-t_{n} ; t_{n}, \mathbf{x}_{t_{n}}\right)
\end{aligned}
$$

where

$$
\varphi(r ; s, \mathbf{x})=\frac{1}{r} \int_{0}^{r} e^{(r-u) \mathbf{L}(s, \mathbf{x})}\left(\mathbf{f}(s, \mathbf{x})+\mathbf{f}_{t}^{\prime}(s, \mathbf{x})(u-s)\right) d u .
$$

The continuous-time $L L$ approximation $\mathbf{y}^{L L}(t)$ on $t \in\left[t_{0}, T\right]$ is defined by concatenating the solutions (44) of said local linear problems starting at $\mathbf{y}^{L L}\left(t_{0}\right)=\mathbf{x}_{0}$ :

$$
\mathbf{y}^{L L}(t)=\mathbf{y}^{L L}\left(t ; t_{n}, \mathbf{y}^{L L}\left(t_{n}\right)\right), \quad t \in \boldsymbol{\Lambda}_{n}, \quad n=0, \ldots, N-1 .
$$

Finally, the $L L$ discretization is defined by evaluating the $L L$ approximation at the discrete times $t=t_{n}$,

$$
\mathbf{y}_{t_{n}}^{L L}=\mathbf{y}^{L L}\left(t_{n}\right), \quad n=0, \ldots, N-1 .
$$

A number of schemes have been proposed for computing the LL discretization (see reviews in [3, [12]). An implementation that consists of computing just one matrix exponential is the following (see [10] for more details). For $t \in \boldsymbol{\Lambda}_{n}$ and any $\mathbf{x}_{t_{n}} \in \mathcal{D}, \mathbf{y}^{L L}\left(t ; t_{n}, \mathbf{x}_{t_{n}}\right)$ is written as

$$
\mathbf{y}^{L L}\left(t ; t_{n}, \mathbf{x}_{t_{n}}\right)=\mathbf{x}_{t_{n}}+\mathbf{v}\left(t ; t_{n}, \mathbf{x}_{t_{n}}\right) .
$$

In turn, $\mathbf{v}\left(t ; t_{n}, \mathbf{x}_{t_{n}}\right)$ can be obtained as a block of the matrix exponential $\exp \left(\left(t-t_{n}\right) \mathbf{C}_{n}\right)$ according to the identity

$$
\left[\begin{array}{ccc}
\mathbf{F}\left(t ; t_{n}, \mathbf{x}_{t_{n}}\right) & \mathbf{b}\left(t ; t_{n}, \mathbf{x}_{t_{n}}\right) \mathbf{v}\left(t ; t_{n}, \mathbf{x}_{t_{n}}\right) \\
0 & 1 & c\left(t ; t_{n}, \mathbf{x}_{t_{n}}\right) \\
0 & 0 & 1
\end{array}\right]=e^{\left(t-t_{n}\right) \mathbf{C}_{n}}
$$


where

$$
\mathbf{C}_{n}=\left[\begin{array}{ccc}
\mathbf{f}_{x}^{\prime}\left(t_{n}, \mathbf{x}_{t_{n}}\right) & \mathbf{f}_{t}^{\prime}\left(t_{n}, \mathbf{x}_{t_{n}}\right) \mathbf{f}\left(t_{n}, \mathbf{x}_{t_{n}}\right) \\
0 & 0 & 1 \\
0 & 0 & 0
\end{array}\right] \in \mathbb{R}^{(d+2) \times(d+2)},
$$

and $\mathbf{F}\left(t ; t_{n}, \mathbf{x}_{t_{n}}\right) \in \mathbb{R}^{d \times d}, \mathbf{b}\left(t ; t_{n}, \mathbf{x}_{t_{n}}\right) \in \mathbb{R}^{d}, c\left(t ; t_{n}, \mathbf{x}_{t_{n}}\right) \in \mathbb{R}$ are certain matrix blocks. Thus, the LL discretization can be obtained as

$$
\mathbf{y}_{t_{n+1}}^{L L}=\mathbf{y}_{t_{n}}^{L L}+\mathbf{v}\left(t_{n+1} ; t_{n}, \mathbf{y}_{t_{n}}^{L L}\right),
$$

where $\mathbf{v}\left(t_{n+1} ; t_{n}, \mathbf{y}_{t_{n}}^{L L}\right)$ is computed through (7) with $t=t_{n+1}$.

A number of algorithms are available to compute the matrix exponential involved in this scheme, e.g. those based on stable Padé approximations with the scaling and squaring method, Schur decomposition, or Krylov subspace methods. The choice of one of them should be based on the size and structure of the Jacobian matrix $\mathbf{f}_{x}^{\prime}([8],[14)$.

\section{Local Linearization-Runge Kutta (LLRK) Methods}

In this Section a modification of the LL method is introduced in order to improve its accuracy while retaining desirable stability properties. Specifically, in order to obtain a better approximation $\mathbf{y}_{n}(t)$ to the solution of the local problem (11) with initial condition $\mathbf{y}_{t_{n}}$ at $t=t_{n}$, consider the addition of a correction term $\mathbf{z}_{n}(t)$ to the LL solution,

$$
\mathbf{y}_{n}(t)=\mathbf{y}^{L L}\left(t ; t_{n}, \mathbf{y}_{t_{n}}\right)+\mathbf{z}_{n}(t), \quad t \in \boldsymbol{\Lambda}_{n}
$$

where $\mathbf{y}^{L L}\left(t ; t_{n}, \mathbf{y}_{t_{n}}\right)$ is defined by (4) .

From the variation of constants formula (see, e.g., [5]) it follows that the solution $\mathbf{x}_{n}(t)$ of (1) starting from $\mathbf{y}_{t_{n}}$ at $t=t_{n}$ can be written as

$$
\mathbf{x}_{n}(t)=\mathbf{y}^{L L}\left(t ; t_{n}, \mathbf{y}_{t_{n}}\right)+\mathbf{r}_{n}(t)
$$

where

$$
\mathbf{r}_{n}(t)=\boldsymbol{\Phi}\left(t ; t_{n}, \mathbf{y}_{t_{n}}\right) \int_{t_{n}}^{t} \boldsymbol{\Phi}^{-1}\left(u ; t_{n}, \mathbf{y}_{t_{n}}\right) \mathbf{M}\left(u, \mathbf{x}_{n}(u) ; t_{n}, \mathbf{y}_{t_{n}}\right) d u
$$

$\mathbf{M}\left(u, \mathbf{x} ; t_{n}, \mathbf{y}_{t_{n}}\right)=\mathbf{f}(u, \mathbf{x})-\left(\mathbf{L}\left(t_{n}, \mathbf{y}_{t_{n}}\right) \mathbf{x}+\mathbf{a}\left(u ; t_{n}, \mathbf{y}_{t_{n}}\right)\right)$, and $\mathbf{L}$, a defined by (3) $)$

By taking derivatives in (10) it is obtained that $\mathbf{r}_{n}(t)$ satisfies the initial-value problem

$$
\mathbf{r}_{n}^{\prime}(t)=\mathbf{g}_{n}\left(t, \mathbf{r}_{n}(t)\right), \quad t \in \boldsymbol{\Lambda}_{n}, \quad \mathbf{r}_{n}\left(t_{n}\right)=0,
$$

where

$$
\mathbf{g}_{n}(t, \mathbf{r})=L\left(t_{n}, \mathbf{y}_{t_{n}}\right) \mathbf{r}+\mathbf{M}\left(t, \mathbf{y}^{L L}\left(t ; t_{n}, \mathbf{y}_{t_{n}}\right)+\mathbf{r} ; t_{n}, \mathbf{y}_{t_{n}}\right) .
$$

Thus, an approximation $\mathbf{z}_{n}(t)$ to $\mathbf{r}_{n}(t)$ can be obtained by solving the initialvalue problem (11) through any extant numerical integrator. In particular, we 
will focus on the approximation $\mathbf{y}_{n}(t)$ obtained from solving (11) by means of an explicit RK method. This will be called a Local Linearization-Runge Kutta (LLRK) approximation. Specifically, the choice of an s-stage explicit RK method with coefficients $\mathbf{c}=\left[c_{i}\right], \mathbf{A}=\left[a_{i j}\right], \mathbf{b}=\left[b_{j}\right]$ (see, [5] ) leads to

$$
\mathbf{z}_{n}(t)=\left(t-t_{n}\right) \sum_{j=1}^{s} b_{j} \mathbf{k}_{j}, \quad t \in \boldsymbol{\Lambda}_{n}
$$

where

$$
\mathbf{k}_{i}=\mathbf{g}_{n}\left(t_{n}+c_{i}\left(t-t_{n}\right),\left(t-t_{n}\right) \sum_{j=1}^{i-1} a_{i j} \mathbf{k}_{j}\right), \quad i=1,2, \ldots s,
$$

Finally, the LLRK discretization is defined by the recursion

$$
\mathbf{y}_{t_{n+1}}=\mathbf{y}^{L L}\left(t_{n+1} ; t_{n}, \mathbf{y}_{t_{n}}\right)+\mathbf{z}_{n}\left(t_{n+1}\right), \quad n=0,1, \ldots, N-1,
$$

starting at $\mathbf{y}_{t_{0}}=\mathbf{x}_{0}$.

When implementing the LLRK method (12), the required evaluations of $\mathbf{y}^{L L}$ $\left(t ; t_{n}, \mathbf{y}_{t_{n}}\right)$ at $t=t_{n+1}$ and $t=t_{n}+c_{i}\left(t_{n+1}-t_{n}\right)$ can be computed by means of (6) -(8). We use the diagonal Padé approximation with the scaling and squaring method 14 to evaluate the matrix exponential involved in these expressions.

It should be noted some points that contribute to decrease the burden of the computation of (12). First, simplifications arise from the fact that $\mathbf{g}_{n}\left(t_{n}, \mathbf{0}\right)=\mathbf{0}$. Second, if (as usually) the Runge Kutta coefficients $c_{i}$ are of the form $c_{i}=m_{i} a$ for some integer numbers $m_{i}$ then (12) can be implemented in terms of a few powers of the same matrix exponential $\exp \left(a h_{n} C_{n}\right)$, where $C_{n}$ is certain matrix.

Given $p \geq 2$, the notation LLRK $p$ will indicate an LLRK method (12) obtained by using an order $p$ RK method to compute $\mathbf{z}_{n}(t)$. Notice that any LLRK $p$ discretization is A-stable because the LL discretization is so, and the former reduces to the latter for linear systems. Furthermore, it can be shown that any LLRK $p$ method has order of convergence $p$ if the needed evaluations of $\mathbf{y}^{L L}\left(. ; t_{n}, \mathbf{y}_{t_{n}}\right)$ are carried out with an error of order $p$.

\section{Numerical Examples}

The following example is taken from [2] in order to illustrate not only convergence issues of the LLRK discretization but also its dynamics around hyperbolic stationary points.

Example 1.

$$
\begin{aligned}
& x_{1}^{\prime}=-2 x_{1}+x_{2}+1-\mu f\left(x_{1}, \lambda\right), \\
& x_{2}^{\prime}=x_{1}-2 x_{2}+1-\mu f\left(x_{2}, \lambda\right),
\end{aligned}
$$

where $f(u, \lambda)=u\left(1+u+\lambda u^{2}\right)^{-1}$. 
For $\mu=15, \lambda=57$, this system has two stable stationary points and one unstable stationary point in the region $0 \leq x_{1}, x_{2} \leq 1$. There is a nontrivial stable manifold for the unstable point which separates the basins of attraction for the two stable points (see [2]).

Figure 1 illustrates the performance of the LLRK3 scheme in this example. For comparison, Figure 1(a) presents the phase portrait obtained by the LL scheme with a very small step-size $\left(h=2^{-13}\right)$, which can be regarded the exact solution for visualization purposes. Figures 1 (b)-(c)-(d) show the phase portraits obtained, respectively, by a third order explicit Runge-Kutta (RK3), the LL and the LLRK3 methods with step-size $h=2^{-2}$.

True Solution

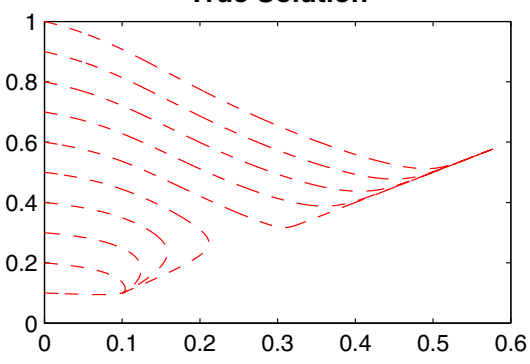

LL Solution

(c)

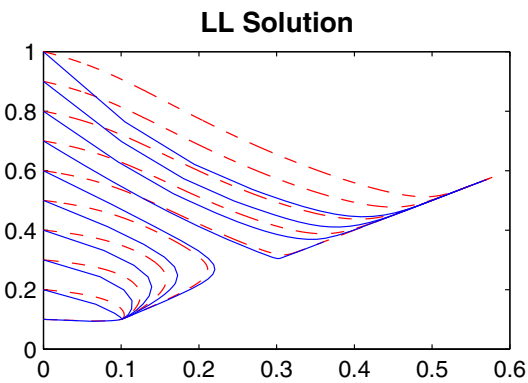

RK3 Solution

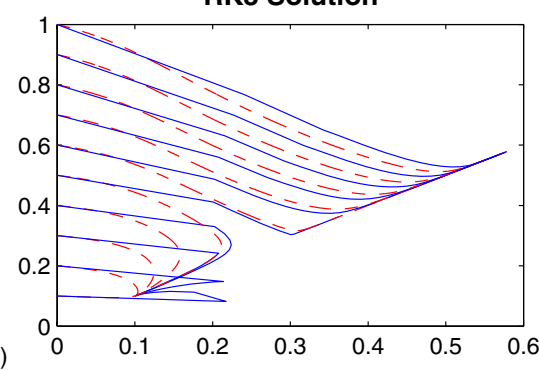

LLRK3 Solution

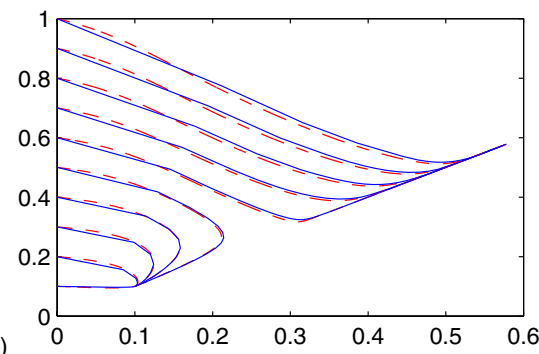

Fig. 1. (Example 1). a) Phase portrait obtained by the LL scheme with a very small step-size, $h=2^{-13}$ (which can be thought of as the exact solution for visualization purposes). b), c), d) Continuous lines show the phase portraits obtained, respectively, by a third order Runge-Kutta (RK3), the LL and the LLRK3 methods with step-size $h=2^{-2}$. For reference, the exact trajectories are also shown in each case as dashed lines.

It can be observed that the RK3 discretization fails to reproduce correctly the phase portrait of the underlying system near one of the point attractors. On the contrary, the exact phase portrait is adequately approximated near both point attractors by the LL and LLRK3 methods, the latter showing better accuracy. Also notice that the RK3 and LL discretizations do not approximate adequately the basins of attraction in the region shown in Figure 1. For instance, RK3 trajectories starting near $(0,0.5)$ and LL trajectories starting near $(0,0.6)$ go 
towards wrong point attractors in comparison with exact trajectories. In contrast, the attracting sets are much better represented by the phase portrait of the LLRK3 method. This demonstrates that, even for moderate values of $p$, the larger accuracy of LLRK $p$ methods in comparison with the LL method can in practice leads to appreciable improvement in dynamical performance.

The next example illustrates the behavior of the LLRK4 method in a wellknown stiff system that is frequently considered to test numerical integrators; namely, the Van der Pol equation (see, e.g., [7]).

Example 2.

$$
\begin{aligned}
& x_{1}^{\prime}=x_{2}, \\
& x_{2}^{\prime}=E\left(\left(1-x_{1}^{2}\right) x_{2}-x_{1}\right),
\end{aligned}
$$

where $E=10^{3}$.

Figure 2 shows the approximations obtained for this example by a fourth order Runge-Kutta (RK4), the LL and the LLRK4 methods for different step sizes. The trajectories start at $(2,0)$. For large step sizes the Runge-Kutta discretizations are explosive due to lack of numerical stability, while the LL approximations do not explode but show poor accuracy. In particular, note that the trajectories of the LL approximation for large and small step sizes have remarkably different
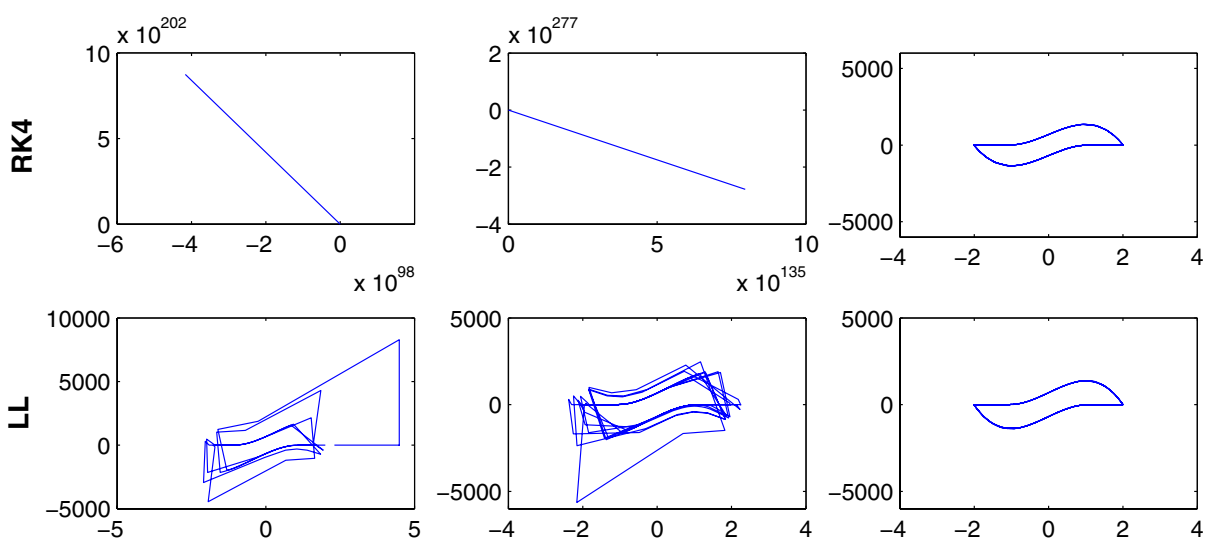

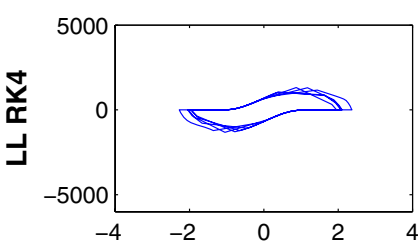

(a)

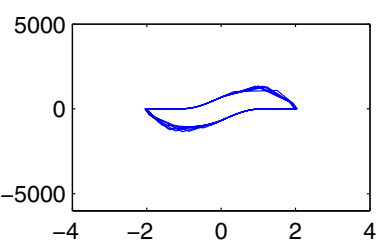

(b)

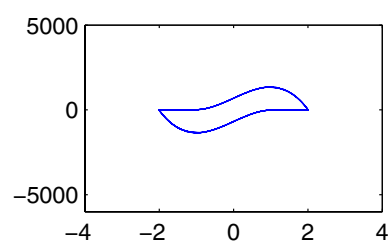

(c)

Fig. 2. (Example 2). Trajectories obtained for the example 2 by means of a fourth order Runge-Kutta (RK4), the LL and the LLRK4 methods for several step sizes: a) $h_{1}=0.00115$, b) $h_{2}=0.00095$, and c) $h_{3}=0.00025$. 
ranges. In contrast, even for large step sizes the LLRK4 method achieves precise reconstructions of the underlying dynamics associated with a limit cycle.

\section{References}

1. Ascher, U. M., Ruuth, S. J. and Spiteri, R. J.: Implicit-Explicit Runge-Kutta methods for time-depending partial differential equations. Appl. Numer. Math. 25 (1995) 151-167

2. Beyn,W. J.: On the numerical approximation of phase portraits near stationary points. SIAM J.Numer. Anal. 24 (1987) 1095-1113

3. Biscay, R. J., De la Cruz, H., Carbonell, F., Ozaki, T. and Jimenez, J. C.: A Higher Order Local Linearization Method for Solving Ordinary Differential Equations. Technical Report, Instituto de Cibernetica, Matematica y Fisica, La Habana. (2005)

4. Bower, J. M. and Beeman, D.: The book of GENESIS: exploring realistic neural models with the general neural simulation system. Springer-Verlag (1995)

5. Butcher, J. C.: The Numerical Analysis of Ordinary Differential Equations. RungeKutta and General Linear Methods. John Wiley \& Sons: Chichester (1987)

6. Carroll, J.: A matricial exponentially fitted scheme for the numerical solution of stiff initial-value problems. Computers Math. Applic. 26 (1993) 57-64.

7. Hairer, E. and Wanner, G.: Solving Ordinary Differential Equations II. Stiff and Differential-Algebraic Problems. Springer-Verlag: Berlin. Third edition (1996)

8. Higham, N. J.: The scaling and squaring method for the matrix exponential revisited. Numerical Analysis Report 452, Manchester Centre for Computational Mathematics. (2004)

9. Hochbruck, M., Lubich, C. and Selhofer, H.: Exponential integrators for large systems of differential equations. SIAM J. Sci. Comput. 19 (1998) 1552-1574

10. Jimenez, J. C.: A Simple Algebraic Expression to Evaluate the Local Linearization Schemes for Stochastic Differential Equations. Appl. Math. Lett. 15 (2002) 775-780

11. Jimenez, J. C., Biscay, R. J., Mora, C. M., and Rodriguez, L. M.: Dynamic properties of the Local Linearization method for initial-valued problems. Appl. Math. Comput. 126 (2002) 63-81

12. Jimenez, J. C. and Carbonell, F.: Rate of convergence of local linearization schemes for initial-value problems. Appl. Math. Comput. 171 (2005) 1282-1295

13. McLachlan, R.I and Quispel, G. R.W.: Splitting methods. Acta Numer. 11 (2002) $341-434$

14. Moler, C. and Van Loan, C. F.: Nineteen dubious ways to compute the exponential of a matrix, twenty-five years later. SIAM Review. 45 (2003) 3-49

15. Ozaki, T.: A bridge between nonlinear time series models and nonlinear stochastic dynamical systems: a local linearization approach. Statist. Sinica. 2 (1992) 113-135

16. Ramos, J. I. and Garcia-Lopez, C. M. (1997).: Piecewise-linearized methods for initial-value problems. Appl. Math. Comput. 82 (1992) 273-302 